\title{
BEMERKUNG ZU MEINER ARBEIT: AUS DER PRAXIS DES PHYSIKALISCH-CHEMISCHEN UNTERRICHTS ${ }^{1}$ ). \\ Von R. Luther.
}

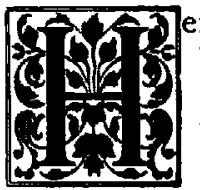

err Geheimrat Nernst macht mich brieflich darauf aufmerksam, dass eine Stelle ${ }^{2}$ ) in der obigen Abhandlung dahin missverstanden werden kann, als habe ich auf einen groben Fehler in der Nernstschen Beweisfahrung hinweisen wollen. Dies ist in keiner Hinsicht der Fall. Es lag mir vielmehr ausschliesslich daran, zu zeigen, dass die Fiktion idealer spezifischer Katalysatoren nicht bloss die Berechnung des Massenwirkungsgesetzes meines Erachtens einfacher und anschaulicher gestaltet, als die Verwendung der von $\mathrm{Ner}$ s $\mathrm{t}$ vorgeschlagenen Hilfsmittel (Kondensation, Ueberfahrung in ein nicht dissociierendes Lösungsmittel), sondern vor allem auch die Arbeit eines beliebigen chemischen Vorganges gedanklich bequem realisierbar erscheinen lässt.

Ich möchte hervorheben, dass meine Abhandlung - wie aus dem Titel und dem ganzen Inhalt hervorgeht — sich ausschliesslich mit pädagogischen Fragen befasste, so dass eine wissenschaftliche Polemik dem Wesen nach gar nicht hineingehört hätte.

Der Einfachheit wegen wiederhole ich meinen Gedankengang etwas ausführlicher in einer Form, die hoffentlich jede Möglichkeit einer Missdeutung ausschliesst.

Ableitung der Reaktionsisotherme und der maximalen Arbeit eines chemischen Vorganges.

Bekanntlich hat van't Hoff ${ }^{3}$ ) zur Ableitung der Reaktionsisotherme, resp. zur isothermen Gewinnung der maximalen Arbeit, welche ein chemischer Vorgang liefern kann, einen Mechanismus mit Kolben und halbdurchlăssigen Wänden erfunden, welcher die nötigen Operationen in sehr anschaulicher Form auszufuhren erlaubt: In gewissen Fallen geht aber die Anschaulichkeit verloren, denn die erforderlichen Operationen mit Additions. Dissociationsvorgängen oder Polymerisationsvorgängen, z. B. $\mathrm{HCl}$ (Gas) $+\mathrm{NH}_{3}$ (Gas) $\rightarrow \mathrm{NH}_{4} \mathrm{Cl}$ (Gas) oder $2 \mathrm{NO}_{2}$ (Gas) $\rightarrow \mathrm{N}_{2} \mathrm{O}_{4}$ (Gas) scheinen im van't Hoffschen Mechanismus im Prinzip nicht realisierbar zu sein. Man müsste nămlich im stande sein, alle Operationen mit den Gasen $\mathrm{NH}_{4} \mathrm{Cl}, \mathrm{NO}_{2}$ und $\mathrm{N}_{2} \mathrm{O}_{4}$ vorzunehmen, ohne dass sie hierbei dissociieren oder sich polymerisieren.

I) Z. f. Elektroch. 12, 97 (1906)

2) 1. c., S. 98 , rechts: Ich glaube . . . belangloser Weise modifiziert.

3) Vergl. Z. f. physikal. Chem. 1, 488 (1887).
Nernst ${ }^{1}$ ) schreibt hierzu: "Ueber dies Bedenken hilft uns aber die Bemerkung hinweg, dass wir die betreffende Molekülgattung nur scbnell genug dem Reaktionsgemische zu entziehen und in einen Zustand zu bringen brauchen, in welchem die Dissociation nicht mehr stattfindet, $z$. B. in einen Zustand starker Kondensation oder in ein nicht dossociierendes Lösungsmittel, wodurch der beschriebene Vorgang in einer fâr das Endresultat gänzlich belanglosen Weise modifiziert wird. Gibt man nur die Möglichkeit $z u$, dass man schnell genug zu operieren vermag, so ist der beschriebene Vorgang in allen Fallen realisierbar, und wir finden das Gesetz der Massenwirkung als ein strenges Postulat der Thermodynamik. " Vorausgesetzt, dass die Ueberfahrung in den Zustand der Kondensation u.s.w. is otherm und reversibel erfolgt, ist dieser gedankliche Kunstgriff selbstverständlich vollkommen hinreichend, um das Massenwirkungsgesetz auf Grundlage der Thermodynamik und der Gesetze idealer Gase und verdünnter Lösungen streng zu beweisen. Hierauf kommt es Nernst bei der Einführung des Kunstgriffes ausschliesslich an, wie aus dem Zusammenhang der zitierten Stelle deutlich hervorgeht.

Dagegen wird mit der Anwendung dieses Kunstgriffes sofort die Anschaulichkeit des Beweises stark verringert, denn erstens ist eine rasche und doch isotherme und reversible Kondensation schwer vorstellbar, dann aber haben wir jetzt gar nicht mehr die Arbeit, die wir ursprunglich berechnen wollten: die Ueberfahrung von Gasen in Gase. Im ersten Beispiel haben wir vielmehr als Endprodukt festen Salmiak, im zweiten Beispiel mussen wir sogar von kọdensiertem $\mathrm{NO}_{2}$ ausgehen und gelangen zu kondensiertem $\mathrm{N}_{2} \mathrm{O}_{4}$. Es tritt mithin zu der Arbeit, die wir ursprünglich berechnen wollten, noch die Kondensationsarbeit hinzu. Auch die gedankliche Realisierung der Arbeit, die der vielbenutzten Gleichung $A=R T \ln K$ zu Grunde liegt, geht verloren, da sich diese Gleichung auf verdannte Zustånde bezieht.

Für diese Seite der Angelegenheit ist die Modifikation, die der Vorgang durch Einführung der Kondensation erleidet, pädagogisch keineswegs belanglos. Erfahrungsgemäss machen nun diese Bedenken den meisten Studenten grosse Schwierigkeiten.

Hier scheint nun die von Nernst am Schlusse des zitierten Passus gemachte Bemerkung zu helfen: "Gibt man nur die Møglichkeit zu, dass man schnell genug zu operieren vermag,

I) Theoret. Chem., 4. Aufl., S. 633 . 
so ist der beschriebene Vorgang in allen Fällen realisierbar." In der 'Tat sieht man sofort ein, dass man durch genügend $\mathrm{ras} \mathrm{ch}$ es Operieren die Gase $\mathrm{NH}_{4} \mathrm{Cl}, \mathrm{NO}_{2}$ oder $\mathrm{N}_{2} \mathrm{O}_{4}$ auch ohne merkliche Dissociation oder Polymerisation komprimieren und dilatieren könnte, denn die Dissociation und Polymerisation erfolgen zwar mit sehr grosser, aber, wie jeder andere Vorgang, keineswegs mit unendlich grosser Geschwindigkeit.

Nun ist man aber mit Recht gewöhnt, sich bei umkehrbaren, isothermen Vorgängen die Volumänderungen langsam vorgehend zu denken. Das erforderliche rasche Operieren widerspricht daher der Anforderung der Umkehrbarkeit und Isothermie.

Gelänge es aber - wenn auch nur gedanklich - die störende Reaktionsgeschwindigkeit so herabzusetzen, dass man langsam operieren kơnnte, so wäre die gedankliche Realisierbarkeit isothermer, reversibler Volumänderungen selbst bei instabilen Stoffen gewährleistet. Dies liesse sich nun erreichen, wenn wir für jeden Vorgang negative ${ }^{1}$ ) oder positive Katalysatoren hätten, die schon in möglichst geringer Konzentration wirksam sein müssten. Mit Hilfe derartiger idealer, positiver und negativer Katalysatoren liessen sich alle Stufen des Vorganges durchaus anschaulich denken; im Gleichgewichtsraum wären hierbei positive, in den instabilen Gasen negative Katalysatoren anzunehmen, und sämtliche Volumänderungen könnten isotherm und reversibel, d. h. langsam vorgenommen werden.

Wenngleich keineswegs für alle Reaktionen derartige Katalysatoren bekannt sind, so gibt es doch eine ganze Reile von Fällen, wo katalytische Reaktionsbeschleunigungen und -hemmungen bereits gefunden sind. Eine Extrapolation auf noch unbekannte Fälle ist ebenso berechtigt oder unberechtigt, wie etwa die Annahme halbdurchlässiger Wände für solche Stoffe, far die derartige Wände bisher noch nicht experimentell realisiert sind. Gerade in dem einen der gewählten Beispiele $\mathrm{HCl}+\mathrm{NH}_{3} \mathrm{NH}_{4} \mathrm{Cl}$ ist nun cin entsprechender Katalysator in Gestalt von Wasserdampf bekannt. Eine Spur Wasserdampf im Gleichgewichtsraum, eine Wand, welche nur $\mathrm{NH}_{4} \mathrm{Cl}$-Dampf, aber keine Spur $\mathrm{H}_{2} \mathrm{O}$-Dampf durchlässt, würden es ermöglichen, dass sämtliche Operationen gedanklich bequem realisierbar erschcinen.

Auch die von Turbaba ${ }^{2}$ ) und Hollmann ${ }^{3}$ ) untersuchte gegenseitige Umwandlung von Acet-

I) Der „Mechanismus" der negativen Katalyse hat mit der Angelegenheit nichts zu tun.

2) Zeitschr. f. physik. Chem. 38, 505 (I9OI).

3) Ebenda 43, 129 (1903). aldehyd und Paraldehyd bei Anwesenheit und Abwesenheit von Katalysatoren bietet ein pädagogisch sehr gutes Beispiel.

Die Einführung der idealen Katalysatoren bei der Berechnung der Arbeit eines beliebigen chemischen Vorganges hat noch insofern eine pädagogische Berechtigung, als wir ja auch experimentell nur solche Vorgănge isotherm und reversibel realisieren können, bei denen die massgebenden Geschwindigkeiten - sei es von Natur, sei es durch Katalysatoren - keine extremen Werte haben. Den Vorgang: $2 \mathrm{NO}_{2}$ $\rightarrow \mathrm{N}_{2} \mathrm{O}_{4}$ konnen wir wegen zu grosser, den Vorgang: $\mathrm{N}_{2}+{ }_{3} \mathrm{H}_{2} \longrightarrow 2 \mathrm{NH}_{3}$ wegen zu kleiner Geschwindigkeit zur Zeit in keiner Weise bei Zimmertemperatur reversibel ausführen.

Die willkürliche Verfügung über die Geschwindigkeit, mit der ein instabiler Zustand dem Gleichgewicht zustrebt, ist übrigens ein Kunstgriff, der im Interesse der Anschaulichkeit auch auf anderen theoretischen Gebieten vielfach benutzt wird, und den man, streng genommen, überhaupt nicht entbehren kann, denn nur so ist es überhaupt möglich, mit den instabilen $\mathrm{Zu}$ ständen reversible Operationen vorzunehmen.

So muss Carnot in seinem Kreisprozess die Geschwindigkeit des Temperaturausgleichs willkürlich durch Wärmeleiter oder durch "vollkommene" Nichtleiter regeln. So wird in der Thermodynamik der Strahlung der Uebergang der instabilen Strahlung in "schwarze" nach Bedarf durch Anwendung theoretisch spiegelnder Wände verzögert oder durch Katalysatoren ( schwarzes Stăubchen") beschleunigt. So müssen in der Elektrostatik "vollkommene" Isolatoren fingiert werden u. s. w.

Auch auf unserem Gebiet kennen wir einen derartigen Fall. Die pådagogisch unentbehrlichen „halbdurchlässigen" Wände differenzieren streng genommen nur die Geschwindigkeit des Stoffdurchtritts. Denn da nach unseren Erfahrungen jeder Stoff in jedem anderen - zum mindesten spurenhaft -- löslich ist, so kann es überhaupt keine vollkommen undurchlässigen Wände geben. Wände können den Stoffausgleich im besten Fall verzögern, sie können diesen Ausgleich selektiv in sehr verschiedenem Masse verzögern (halbdurchlässige Wände), theoretisch hindern können sie ihn nie.

Aebnliche Verhältnisse finden sich überall, nur sind meist die implicite gemachten Annahmen uns so geläufig, dass wir sie gar nicht mehr als Annahmen erkennen.

Leipzig, Juli 1907 .

(Eingegangen: 2. August.) 